\title{
Some Optimal Error Estimates for Piecewise Linear Finite Element Approximations
}

\author{
By Rolf Rannacher and Ridgway Scott
}

\begin{abstract}
It is shown that the Ritz projection onto spaces of piecewise linear finite elements is bounded in the Sobolev space, $W_{p}^{1}$, for $2 \leqslant p \leqslant \infty$. This implies that for functions in $\dot{W}_{p}^{1} \cap W_{p}^{2}$ the error in approximation behaves like $O(h)$ in $W_{p}^{1}$, for $2 \leqslant p \leqslant \infty$, and like $O\left(h^{2}\right)$ in $L_{p}$, for $2 \leqslant p<\infty$. In all these cases the additional logarithmic factor previously included in error estimates for linear finite elements does not occur.
\end{abstract}

1. Introduction and Results. Let $\Omega$ be a convex polygonal domain in $\mathbf{R}^{2}$, and let $\pi_{h}=\{K\}, 0<h \leqslant h_{0}<1$, be finite triangulations of $\Omega$ such that the usual regularity condition is satisfied:

(T) The triangles $K \in \pi_{h}$ meet only in entire common sides or in vertices. Each triangle $K \in \pi_{h}$ contains a circle of radius $c_{1} h$ and is contained in a circle of radius $c_{2} h$, where the constants $c_{1}>0$ and $c_{2}<\infty$ do not depend on $K$ or $h$.

Corresponding to $\pi_{h}$, we define the finite dimensional subspace $S_{h} \subset \stackrel{\circ}{W}_{\infty}^{1}$ by

$$
S_{h}=\left\{v_{h} \in \stackrel{\circ}{W}_{\infty}^{1}: v_{h} \text { is linear on each } K \in \pi_{h}\right\},
$$

and the Ritz projection $R_{h}: \stackrel{\circ}{W}_{2}^{1} \rightarrow S_{h}$ by

$$
\left(\nabla R_{h} u, \nabla \varphi_{h}\right)=\left(\nabla u, \nabla \varphi_{h}\right) \quad \forall \varphi_{h} \in S_{h} .
$$

Here $L_{p}$ and $W_{p}^{m}, 1 \leqslant p \leqslant \infty, m \in \mathbf{N}$, are the Lebesgue and Sobolev spaces on $\Omega$ provided with the usual norms $\|\cdot\|_{p}$ and $\|\cdot\|_{m, p}$, respectively. $\dot{W}_{p}^{1}$ is the subspace of those functions in $W_{p}^{1}$ which vanish on the boundary in the generalized sense. The inner product of $L_{2}$ is denoted by $(\cdot, \cdot)$. Finally, by $c$ we mean a generic positive constant which may vary with the context but is always independent of $h$.

Under assumption (T), we have the well-known mean-square-error estimates

$$
\left\|u-R_{h} u\right\|_{k, 2} \leqslant c h^{2-k}\|u\|_{2,2}, \quad k=0,1,
$$

and the uniform-error estimates (see [4], [8], [6], [1], [7])

$$
\left\|u-R_{h} u\right\|_{k, \infty} \leqslant c h^{2-k} \ln \frac{1}{h}\|u\|_{2, \infty}, \quad k=0,1 .
$$

From (1.2) and (1.3) one may conclude, by an interpolation argument, that for $2 \leqslant p<\infty$ the $L_{p}$ error behaves like (see [8])

$$
\left\|u-R_{h} u\right\|_{p} \leqslant c h^{2}\left(\ln \frac{1}{h}\right)^{1-2 / p}\|u\|_{2, p} .
$$

Received March 10, 1981

1980 Mathematics Subject Classification. Primary 65N30.

Key words and phrases. Maximum norm estimates, finite element methods.

(C)1982 American Mathematical Society $0025-5718 / 81 / 0000-0124 / \$ 02.50$ 
It has been considered as a challenge from the beginning to remove the logarithmic factors in (1.3) and (1.4). This, in particular, since one can show that for higher than second order finite elements these estimates hold without the logarithm; see [5], [8]. Also, for any function $u \in \stackrel{\circ}{W}_{p}^{1} \cap W_{p}^{2}, 1 \leqslant p \leqslant \infty$, the natural piecewise linear interpolant $I_{h} u \in S_{h}$ is well defined and satisfies

$$
\left\|u-I_{h} u\right\|_{k, p} \leqslant c h^{2-k}\|u\|_{2, p}, \quad k=0,1 .
$$

For the case of linear finite elements, Fried [2] has recently published an example* based on radial symmetry which indicates that (in two and three dimensions) at least the pointwise estimate

$$
\left\|u-R_{h} u\right\|_{\infty} \leqslant c h^{2} \ln \frac{1}{h}\|u\|_{2, \infty}
$$

may be of optimal order. However, this leaves the question open whether the $L_{p}$ estimate (1.4) is optimal. In the present paper we shall give an answer to this question for the model situation considered here which is based on the following stability result:

THEOREM. Under assumption (T) the Ritz projection $R_{h}$ is stable in $\stackrel{\circ}{p}_{p}^{1}$ for $2 \leqslant p \leqslant \infty$, namely

$$
\left\|R_{h} u\right\|_{1, p} \leqslant c\|u\|_{1, p} .
$$

The proof of the theorem will be given in the next two sections. One of its consequences is the following

COROLlARY. Under assumption (T), for any function $u \in \stackrel{\circ}{W}_{p}^{1} \cap W_{p}^{2}$ there holds

$$
\begin{array}{rlrl}
\left\|u-R_{h} u\right\|_{1, p} \leqslant c h\|u\|_{2, p}, & & 2 \leqslant p \leqslant \infty \\
\left\|u-R_{h} u\right\|_{p} \leqslant c_{p} h^{2}\|u\|_{2, p}, & 2 \leqslant p<\infty .
\end{array}
$$

Proof. We apply (1.7) for $u-I_{h} u$ and observe that $R_{h} \equiv$ id on $S_{h}$ to obtain

$$
\left\|R_{h} u-I_{h} u\right\|_{1, p} \leqslant c\left\|u-I_{h} u\right\|_{1, p}, \quad 2 \leqslant p \leqslant \infty .
$$

Then, the approximation estimate (1.5) implies (1.8).

To prove (1.9), we use a duality argument. Let $p \in[2, \infty)$, so that $q \equiv p /(p-1)$ $\in(1,2]$. On the convex polygonal domain $\Omega$, the Laplacian is a homeomorphism from $\mathscr{b}_{q}^{1} \cap W_{q}^{2}$ onto $L_{q}, 1<q \leqslant 2$; see [3]. Hence there is a $v \in \dot{W}_{q}^{1} \cap W_{q}^{2}$ satisfying

$$
-\Delta v=\operatorname{sgn}\left(u-R_{h} u\right)\left|u-R_{h} u\right|^{p-1} \text { in } \Omega
$$

and

$$
\|v\|_{2, q} \leqslant c\|\Delta v\|_{q}=c\left\|u-R_{h} u\right\|_{p}^{p-1}
$$

Using now (1.1), Hölder's inequality, (1.5), (1.8), and (1.10), we find

$$
\begin{aligned}
\left\|u-R_{h} u\right\|_{p}^{p} & =\left(\nabla\left(u-R_{h} u\right), \nabla\left(v-I_{h} v\right)\right) \\
& \leqslant\left\|u-R_{h} u\right\|_{1, p}\left\|v-I_{h} v\right\|_{1, q} \leqslant\left\|u-R_{h} u\right\|_{1, p} c h\|v\|_{2, q} \\
& \leqslant c h^{2}\|u\|_{2, p}\|v\|_{2, q} \leqslant c h^{2}\|u\|_{2, p}\left\|u-R_{h} u\right\|_{p}^{p-1} \text {. Q.E.D. }
\end{aligned}
$$

* This example had already appeared in Jespersen [9], however. 
We remark on some extensions of our results. The proof of the theorem and to a large extent also that of its corollary make use of the fact that the Laplacian considered as a mapping

$$
\Delta: \stackrel{\circ}{W}_{p}^{1} \cap W_{p}^{2} \rightarrow L_{p}
$$

is a homeomorphism for $p \in(1,2+\alpha$, where $\alpha$ is some arbitrarily small but positive number. This is certainly true on a domain with smooth boundary, say $\partial \Omega \in C^{2}$, for all $\alpha>0$, and it is known also for convex polygonal domains (see [3]) where $\alpha$ depends on the size of the maximum inner angle, $\omega<\pi$. Our results extend to more general second order elliptic operators as long as the corresponding mapping (1.12) is a homeomorphism. In the case of a curved boundary the proofs become more involved due to the approximation of $\Omega$ by polygonal domains $\Omega_{h}$. In the case that $\partial \Omega$ is smooth one can show that for all $p \in(1, \infty]$ the following refined estimate holds:

$$
\left\|R_{h} u\right\|_{1, p} \leqslant c\left\{\|u\|_{1, p ; \Omega_{h}}+h^{1-1 / p}\|u\|_{1, p ; \Omega \backslash \Omega_{h}}\right\} .
$$

From that estimate one can again draw the conclusions (1.8) and (1.9), now valid for all $p \in(1, \infty]$ and $p \in(1, \infty)$, respectively. The results for $1<p<2$ are proved via a duality argument that makes use of elliptic regularity results that are not generally valid for nonsmooth boundaries.

2. Proof of the Theorem. Notation and techniques are similar to those used in [1]. However, the key difference is in the type of Green's function employed. The basic technique used in several papers is to reduce to the problem of estimating the error $g-R^{h} g$ in approximating the solution of

$$
-\Delta g=\delta \text { in } \Omega
$$

where $\delta$ is the Dirac $\delta$-function or some approximation to it. The difficulty is that, with piecewise linear approximation, the error $g-R^{h} g$ contains a logarithmic factor. For example, it was noted in [8] that $0<c^{-1} \leqslant h^{-1}\left(\ln h^{-1}\right)^{-1}\left\|g-R^{h} g\right\|_{1,1} \leqslant c$ as $h \rightarrow 0$. The reason is that the smoothness of $g$ is such that piecewise linears fail to afford optimal approximation (whereas higher degree piecewise polynomials would yield an approximation rate devoid of the logarithmic factor). The remedy here is to consider instead a "derivative" Green's function, satisfying

$$
-\Delta g=\frac{\partial \delta}{\partial x_{i}} \quad \text { in } \Omega
$$

(for each $i=1,2$ ). Now $g$ is more singular, and piecewise linears afford optimal approximation, albeit at a slower rate. We now turn to the details.

Let $u \in \dot{W}_{p}^{1}, 2 \leqslant p \leqslant \infty$, be given. We pick any point $z \in \Omega$ contained in the interior of some triangle $K_{z} \in \pi_{h}$, and denote by $\partial$ any of the operators $\partial / \partial x_{i}$, $i=1,2$. Because of assumption (T), there is a function $\delta_{z} \in C_{0}^{\infty}\left(K_{z}\right)$ such that

$$
\int \delta_{z} d x=1, \quad\left|\nabla_{k} \delta_{z}\right| \leqslant c h^{-2-k}, \quad k=0,1, \ldots,
$$

where the constant $c$ does not depend on $z$ or $h$. Here $\nabla_{k} \delta_{z}$ denotes the tensor of derivatives of $\delta_{z}$ of order $k$. Then, by construction,

$$
\partial \varphi_{h}(z)=\left(\partial \varphi_{h}, \delta_{z}\right) \quad \forall \varphi_{h} \in S_{h} .
$$


Correspondingly, we define $g_{z} \in \stackrel{\circ}{W}_{2}^{1}$ by

$$
\left(\nabla g_{z}, \nabla \varphi\right)=\left(\delta_{z}, \partial \varphi\right) \quad \forall \varphi \in \stackrel{\circ}{W}_{2}^{1} .
$$

Clearly, $g_{z}$ is a regularized derivative of the Green's function of the Laplacian on $\Omega$. Using this notation, we have

$$
\begin{aligned}
\partial R_{h} u(z) & =\left(\nabla R_{h} u, \nabla g_{z}\right)=\left(\nabla u, \nabla R_{h} g_{z}\right) \\
& =\left(\partial u, \delta_{z}\right)-\left(\nabla u, \nabla\left(g_{z}-R_{h} g_{z}\right)\right) .
\end{aligned}
$$

We introduce the weight function

$$
\sigma_{z}(x)=\left(|x-z|^{2}+\kappa^{2} h^{2}\right)^{1 / 2}, \quad \kappa \geqslant 1,
$$

where the parameter $\kappa$ will be chosen appropriately large, $\kappa \geqslant \kappa_{*} \geqslant 1$, but independent of $h$ ! We note that from now on the generic constant $c$ is also independent of $\kappa$ and $z \in \Omega$, and of the parameter $\alpha \in(0,1]$ introduced below.

Suppose temporarily that $p<\infty$. Applying Hölder's inequality to the terms in (2.4), we obtain for any $\alpha \in(0,1]$ that

$$
\begin{aligned}
& \left|\left(\nabla u, \nabla\left(g_{z}-R_{h} g_{z}\right)\right)\right| \\
& \leqslant\left(\int \sigma_{z}^{-2-\alpha}|\nabla u|^{p} d x\right)^{1 / p}\left(\int \sigma_{z}^{-2-\alpha} d x\right)^{(p-2) / 2 p}\left(\int \sigma_{z}^{2+\alpha}\left|\nabla\left(g_{z}-R_{h} g_{z}\right)\right|^{2} d x\right)^{1 / 2} \\
& \leqslant c\left(\alpha^{-1} h^{-\alpha}\right)^{(p-2) / 2 p} M_{h}\left(\int \sigma_{z}^{-2-\alpha}|\nabla u|^{p} d x\right)^{1 / p},
\end{aligned}
$$

where

$$
M_{h}=\max _{z \in \Omega}\left(\int \sigma_{z}^{2+\alpha}\left|\nabla\left(g_{z}-R_{h} g_{z}\right)\right|^{2} d x\right)^{1 / 2} .
$$

Furthermore,

$$
\begin{aligned}
\left|\left(\partial u, \delta_{z}\right)\right| & \leqslant\left(\int_{K_{z}}|\partial u|^{p} d x\right)^{1 / p}\left(\int_{K_{z}}\left|\delta_{z}\right|^{p /(p-1)} d x\right)^{(p-1) / p} \\
& \leqslant c h^{-2 / p}\left(\int_{K_{z}}|\nabla u|^{p} d x\right)^{1 / p}
\end{aligned}
$$

We apply the above estimates with (2.4), raise to the $p$ th power and integrate with respect to $z \in \Omega$ to obtain

$$
\begin{aligned}
\left\|\partial R_{h} u\right\|_{p} \leqslant & c\left(h^{-2} \iint_{K_{z}}|\nabla u|^{p} d x d z\right)^{1 / p} \\
& +c\left(\alpha^{-1} h^{-\alpha}\right)^{(p-2) / 2 p} M_{h}\left(\iint \sigma_{z}^{-2-\alpha}|\nabla u|^{p} d x d z\right)^{1 / p} .
\end{aligned}
$$

Thus, by interchanging integration, we find

$$
\left\|\partial R_{h} u\right\|_{p} \leqslant c\|\nabla u\|_{p}\left(1+\alpha^{-1 / 2} h^{-\alpha / 2} M_{h}\right),
$$

where the constant $c$ is obviously independent of $p$. Estimate (2.6) is also easily seen to hold for $p=\infty$ using the above techniques. Now, to prove the assertion of the 
theorem, we have to show that

$$
M_{h}=\max _{z \in \Omega}\left(\int \sigma_{z}^{2+\alpha}\left|\nabla\left(g_{z}-R_{h} g_{z}\right)\right|^{2} d x\right)^{1 / 2} \leqslant c_{\alpha} h^{\alpha / 2},
$$

for a proper choice of $\alpha \in(0,1]$.

To prove (2.7), we need some preparations. From now on, we drop the subscript $z$ and simply write $\sigma, g$ for $\sigma_{z}, g_{z}$. The weight function $\sigma$ satisfies

$$
\left|\nabla_{k} \sigma\right| \leqslant c \sigma^{1-k} \leqslant c(\kappa h)^{1-k}, \quad k=0,1,2, \ldots
$$

Here $\nabla_{k} \sigma$ denotes the tensor of $k$ th order derivatives of $\sigma$. Moreover, for $\kappa \geqslant \kappa_{1}$ sufficiently large, one has that (see [5])

$$
\max _{K \in \pi_{h}}\left[\max _{x \in K} \sigma(x) / \min _{x \in K} \sigma(x)\right] \leqslant c
$$

holds uniformly for $z \in \Omega$. For any function $v \in \stackrel{\circ}{W}_{2}^{1} \cap\left[\Pi_{K \in \pi_{h}} W_{2}^{2}(K)\right]$ the natural piecewise linear interpolant $I_{h} v \in S_{h}$ is well defined and satisfies

$$
\left\|\nabla\left(v-I_{h} v\right)\right\|_{2 ; K} \leqslant c h\left\|\nabla_{2} v\right\|_{2 ; K}, \quad K \in \pi_{h} .
$$

Combining (2.10) and (2.9), one easily sees that the following holds:

$$
\int \sigma^{\beta}\left|\nabla\left(v-I_{h} v\right)\right|^{2} d x \leqslant c h^{2} \int^{\prime} \sigma^{\beta}\left|\nabla_{2} v\right|^{2} d x
$$

where the abbreviation used is

$$
\int^{\prime} \cdots d x=\sum_{K \in \pi_{h}} \int_{K} \cdots d x
$$

To prove (2.7), we set $\psi=\sigma^{2+\alpha}\left(g-R_{h} g\right)$ and we use (1.1) to obtain

$$
\begin{aligned}
\int \sigma^{2+\alpha} \mid \nabla & \left.\left(g-R_{h} g\right)\right|^{2} d x \\
& =\int \nabla\left(g-R_{h} g\right) \nabla\left(\psi-I_{h} \psi\right) d x+\frac{1}{2} \int \Delta \sigma^{2+\alpha}\left(g-R_{h} g\right)^{2} d x .
\end{aligned}
$$

Thus,

$$
\begin{aligned}
& \int \sigma^{2+\alpha}\left|\nabla\left(g-R_{h} g\right)\right|^{2} d x \\
& \quad \leqslant \int \sigma^{-2-\alpha}\left|\nabla\left(\psi-I_{h} \psi\right)\right|^{2} d x+c \int \sigma^{\alpha}\left(g-R_{h} g\right)^{2} d x .
\end{aligned}
$$

From (2.11), we get by a simple calculation that

$$
\begin{aligned}
\int \sigma^{-2-\alpha} \mid & \left.\nabla\left(\psi-I_{h} \psi\right)\right|^{2} d x \leqslant c h^{2} \int \sigma^{2+\alpha}\left|\nabla_{2} g\right|^{2} d x \\
& +c \kappa^{-2}\left(\int \sigma^{2+\alpha}\left|\nabla\left(g-R_{h} g\right)\right|^{2} d x+\int \sigma^{\alpha}\left(g-R_{h} g\right)^{2} d x\right) .
\end{aligned}
$$

We insert this estimate into (2.12) and find that, for $\kappa \geqslant \kappa_{2}$ sufficiently large,

$$
\int \sigma^{2+\alpha}\left|\nabla\left(g-R_{h} g\right)\right|^{2} d x \leqslant c h^{2} \int \sigma^{2+\alpha}\left|\nabla_{2} g\right|^{2} d x+c \int \sigma^{\alpha}\left(g-R_{h} g\right)^{2} d x .
$$


To handle the second term on the right side of (2.13), we employ a duality argument in weighted norms. For fixed $h$, let $v \in \dot{W}_{2}^{1}$ be the solution of the auxiliary problem

$$
-\Delta v=\sigma^{\alpha}\left(g-R_{h} g\right) \text { in } \Omega .
$$

Since $\Omega$ is convex, it is guaranteed that $v \in W_{2}^{2}$. Moreover, in Section 3 we shall show that the following weighted a priori estimate holds for all $v \in \dot{W}_{2}^{1}(\Omega)$ such that $\Delta v \in \stackrel{\circ}{W}_{2}^{1}(\Omega)$ :

$$
\int \sigma^{-2-\alpha}\left|\nabla_{2} v\right|^{2} d x \leqslant c \alpha^{-1}(\kappa h)^{-2} \int \sigma^{2-\alpha}|\nabla \Delta v|^{2} d x
$$

Consequently,

$$
\begin{aligned}
\int \sigma^{-2-\alpha} \mid & \left.\nabla_{2} v\right|^{2} d x \\
& \leqslant c \alpha^{-1}(\kappa h)^{-2} \int\left\{\sigma^{2+\alpha}\left|\nabla\left(g-R_{h} g\right)\right|^{2}+\sigma^{\alpha}\left(g-R_{h} g\right)^{2}\right\} d x .
\end{aligned}
$$

Using (2.14), we have

$$
\begin{aligned}
& \int \sigma^{\alpha}\left(g-R_{h} g\right)^{2} d x=\int \nabla\left(g-R_{h} g\right) \cdot \nabla\left(v-I_{h} v\right) d x \\
& \leqslant\left(\int \sigma^{2+\alpha}\left|\nabla\left(g-R_{h} g\right)\right|^{2} d x\right)^{1 / 2}\left(\int \sigma^{-2-\alpha}\left|\nabla\left(v-I_{h} v\right)\right|^{2} d x\right)^{1 / 2} .
\end{aligned}
$$

Then, by (2.11) and (2.16), choosing $\kappa \geqslant \kappa_{3}$ sufficiently large yields

$$
\int \sigma^{\alpha}\left(g-R_{h} g\right)^{2} d x \leqslant c(\alpha \kappa)^{-1} \int \sigma^{2+\alpha}\left|\nabla\left(g-R_{h} g\right)\right|^{2} d x .
$$

We insert (2.17) into (2.13) and choose again $\kappa \geqslant \kappa_{4}(\alpha)$ sufficiently large to obtain

$$
\int \sigma^{2+\alpha}\left|\nabla\left(g-R_{h} g\right)\right|^{2} d x \leqslant c h^{2} \int \sigma^{2+\alpha}\left|\nabla_{2} g\right|^{2} d x .
$$

Thus, we have reduced the proof of (2.7) to an a priori estimate of the form

$$
\int \sigma^{2+\alpha}\left|\nabla_{2} g\right|^{2} d x \leqslant c_{\alpha} h^{\alpha-2} .
$$

This estimate, however, is an obvious consequence of the a priori estimate

$$
\int \sigma^{2+\alpha}\left|\nabla_{2} g\right|^{2} d x \leqslant c \int \sigma^{2+\alpha}(\partial \delta)^{2} d x+c \alpha^{-1}(\kappa h)^{-2} \int \sigma^{2+\alpha} \delta^{2} d x,
$$

which will be proven in Section 3 , for $0<\alpha \leqslant \alpha_{\Omega}$ sufficiently small.

3. Some Weighted A Priori Estimates. Let functions $f \in \dot{W}_{2}^{1}$ and $b \in\left[\dot{W}_{2}^{1}\right]^{2}$ be given, and let $v \in \dot{W}_{2}^{1}$ be such that

$$
-\Delta v=f+\operatorname{div} b \quad \text { in } \Omega .
$$

If $\sigma=\left(|x-z|^{2}+\zeta^{2}\right)^{1 / 2}$ is the weight function introduced in Section 2 , then we have the following

LEMMA. For any convex polygonal domain $\Omega$, there exists an $\alpha_{\Omega} \in(0,1]$ such that for all parameter values $\alpha \in\left(0, \alpha_{\Omega}\right]$ the following a priori estimates hold,

(i) if $f \equiv 0$ :

$$
\int \sigma^{2+\alpha}\left|\nabla_{2} v\right|^{2} d x \leqslant c \int \sigma^{2+\alpha}|\operatorname{div} b|^{2} d x+c \alpha^{-1} \zeta^{-2} \int \sigma^{2+\alpha}|b|^{2} d x,
$$


(ii) if $b \equiv 0$ :

$$
\int \sigma^{-2-\alpha}\left|\nabla_{2} v\right|^{2} d x \leqslant c \alpha^{-1} \zeta^{-2} \int \sigma^{2-\alpha}|\nabla f|^{2} d x
$$

Proof. (i) To prove (3.2), we estimate

$$
\int \sigma^{2+\alpha}\left|\nabla_{2} v\right|^{2} d x \leqslant \int\left|\nabla_{2}\left[\sigma^{1+\alpha / 2} v\right]\right|^{2} d x+c \int\left\{\sigma^{\alpha}|\nabla v|^{2}+\sigma^{\alpha-2} v^{2}\right\} d x .
$$

Since $\Omega$ is convex, we have the standard $L_{2}$ a priori estimate

$$
\|w\|_{2,2} \leqslant c\|\Delta w\|_{2}, \quad w \in \stackrel{\circ}{W}_{2}^{1} \cap W_{2}^{2} .
$$

Applying this to $\sigma^{1+\alpha / 2} v$, we find by a simple calculation that

$$
\int \sigma^{2+\alpha}\left|\nabla_{2} v\right|^{2} d x \leqslant c \int \sigma^{2+\alpha}|\operatorname{div} b|^{2} d x+c \int\left\{\sigma^{\alpha}|\nabla v|^{2}+\sigma^{\alpha-2} v^{2}\right\} d x
$$

Furthermore,

$$
\int \sigma^{\alpha}|\nabla v|^{2} d x=\int \nabla v \nabla\left(\sigma^{\alpha} v\right) d x+\frac{1}{2} \int \Delta \sigma^{\alpha} v^{2} d x
$$

and hence, using (3.1),

$$
\int \sigma^{\alpha}|\nabla v|^{2} d x \leqslant c \int \sigma^{2+\alpha}|\operatorname{div} b|^{2} d x+c \int \sigma^{\alpha-2} v^{2} d x
$$

Combining (3.5) with (3.4), we arrive at

$$
\int \sigma^{2+\alpha}\left|\nabla_{2} v\right|^{2} d x \leqslant c \int \sigma^{2+\alpha}|\operatorname{div} b|^{2} d x+c \int \sigma^{\alpha-2} v^{2} d x
$$

Next, we apply Hölder's inequality to obtain

$$
\begin{aligned}
\int \sigma^{\alpha-2} v^{2} d x & \leqslant\left(\int \sigma^{-2-\alpha} d x\right)^{(2-\alpha) /(2+\alpha)}\|v\|_{(2+\alpha) / \alpha}^{2} \\
& \leqslant c\left(\alpha^{-1} \zeta^{-\alpha}\right)^{(2-\alpha) /(2+\alpha)}\|v\|_{(2+\alpha) / \alpha}^{2} .
\end{aligned}
$$

We have already noted that the Laplacian is a homeomorphism from $\stackrel{\circ}{W}_{q}^{1} \cap W_{q}^{2}$ onto $L_{q}$ for all $q \in(1,2]$. Hence, there is a $w \in \stackrel{\circ}{W}_{(2+\alpha) / 2}^{1} \cap W_{(2+\alpha) / 2}^{2}$ satisfying

$$
-\Delta w=\operatorname{sgn}(v)|v|^{2 / \alpha} \text { in } \Omega,
$$

and

$$
\|w\|_{2,(2+\alpha) / 2} \leqslant c\|\Delta w\|_{(2+\alpha) / 2} \text {. }
$$

Then, we have via Hölder's inequality, Sobolev's inequality, and (3.8) that

$$
\begin{aligned}
\|v\|_{(2+\alpha) / \alpha}^{(2+\alpha) / \alpha} & =(\nabla v, \nabla w)=(b, \nabla w) \leqslant\|b\|_{(4+2 \alpha) /(2+3 \alpha)}\|w\|_{1,(4+2 \alpha) /(2-\alpha)} \\
& \leqslant c\|b\|_{(4+2 \alpha) /(2+3 \alpha)}\|w\|_{2,(2+\alpha) / 2} \leqslant c\|b\|_{(4+2 \alpha) /(2+3 \alpha)}\|v\|_{(2+\alpha) / \alpha}^{2} .
\end{aligned}
$$

Thus, we obtain

$$
\|v\|_{(2+\alpha) / \alpha} \leqslant c\|b\|_{(4+2 \alpha) /(2+3 \alpha)} .
$$


Now, again by Hölder's inequality,

$$
\begin{aligned}
\|b\|_{(4+2 \alpha) /(2+3 \alpha)} & \leqslant\left(\int \sigma^{2+\alpha}|b|^{2} d x\right)^{1 / 2}\left(\int \sigma^{-[2+\alpha]^{2} / 2 \alpha} d x\right)^{\alpha /(2+\alpha)} \\
& \leqslant c \zeta^{-\left(4+\alpha^{2}\right) /(4+2 \alpha)}\left(\int \sigma^{2+\alpha}|b|^{2} d x\right)^{1 / 2}
\end{aligned}
$$

Combining the estimates (3.10)-(3.7), we obtain that for $\alpha \in(0,1]$

$$
\int \sigma^{\alpha-2} v^{2} d x \leqslant c \alpha^{-1} \zeta^{-2} \int \sigma^{2+\alpha}|b|^{2} d x
$$

This together with (3.6) proves the estimate (3.2) for the choice $\alpha_{\Omega}=1$.

(ii) To prove (3.3), we apply Hölder's inequality as follows:

$$
\begin{aligned}
\int \sigma^{-2-\alpha}\left|\nabla_{2} v\right|^{2} d x & \leqslant\left(\int \sigma^{-(2+\alpha) / \alpha} d x\right)^{\alpha}\left\|\nabla_{2} v\right\|_{2 /(1-\alpha)}^{2} \\
& \leqslant c \zeta^{\alpha-2}\|v\|_{2,2 /(1-\alpha)}^{2} .
\end{aligned}
$$

Above, we have noted that the Laplacian is a homeomorphism from $\stackrel{\circ}{W}_{2 /(1-\alpha)}^{1} \cap$ $W_{2 /(1-\alpha)}^{2}$ onto $L_{2 /(1-\alpha)}$ for $\alpha \in\left(0, \alpha_{\Omega}\right]$, where $1>\alpha_{\Omega}>0$ is determined by the maximum inner angle of $\Omega$. Thus, for $\alpha \in\left(0, \alpha_{\Omega}\right]$, we have that

$$
\|v\|_{2,2 /(1-\alpha)} \leqslant c\|\Delta v\|_{2 /(1-\alpha)} .
$$

By Sobolev's inequality combined with Poincaré's inequality (notice that $\Delta v \in \stackrel{\circ}{W}_{2}^{1}$ ),

$$
\|\Delta v\|_{2 /(1-\alpha)} \leqslant c\|\nabla \Delta v\|_{2 /(2-\alpha)} .
$$

We apply again Hölder's inequality to obtain

$$
\begin{aligned}
\|\nabla \Delta v\|_{2 /(2-\alpha)} & \leqslant\left(\int \sigma^{-(2-\alpha) /(1-\alpha)} d x\right)^{(1-\alpha) / 2}\left(\int \sigma^{2-\alpha}|\nabla \Delta v|^{2} d x\right)^{1 / 2} \\
& \leqslant c \alpha^{-1 / 2 \zeta^{-\alpha / 2}}\left(\int \sigma^{2-\alpha}|\nabla \Delta v|^{2} d x\right)^{1 / 2} .
\end{aligned}
$$

Combining the estimates (3.15)-(3.12), we finally reach the desired estimate

$$
\int \sigma^{-2-\alpha}\left|\nabla_{2} v\right|^{2} d x \leqslant c \alpha^{-1} \zeta^{-2} \int \sigma^{2-\alpha}|\nabla \Delta v|^{2} d x
$$

valid for $\alpha \in\left(0, \alpha_{\Omega}\right]$. Q.E.D.

Institut für Angewandte Mathematik

Universität Erlangen-Nürnberg

8520 Erlangen, West Germany

Department of Mathematics

University of Michigan

Ann Arbor, Michigan 48109

1. J. Frehse \& R. RanNaCher, "Eine $L^{1}$-Fehlerabschätzung für diskrete Grundlösungen in der Methode der finiten Elemente," Finite Elemente, J. Frehse et al., eds., Bonn. Math. Schrift., No. 89, 1976, pp. 92-114.

2. I. FriED, "On the optimality of the pointwise accuracy of the finite element solution," Internat. $J$. Numer. Methods Engrg., v. 15, 1980, pp. 451-456.

3. P. GRISVARD, "Behavior of solutions of an elliptic boundary value problem in polygonal or polyhedral domains," Numerical Solution of Partial Differential Equations-III (Synspade 1975) (B. Hubbard, ed.), Academic Press, New York, 1976, pp. 207-274. 
4. F. NATTERER, “Über die punktweise Konvergenz finiter Elemente,” Numer. Math., v. 25, 1975, pp. 67-77.

5. J. A. Nitsche, $L^{\infty}$-Convergence of Finite Element Approximation, Second Conf. on Finite Elements, Rennes, France, 1975.

6. J. NitSCHE, " $L_{\infty}$-convergence of finite element approximations," Mathematical Aspects of Finite Element Methods (I. Galligani and E. Magenes, eds.), Lecture Notes in Math., vol. 606, Springer-Verlag, Berlin, 1977, pp. 261-274.

7. A. H. SChatz \& L. B. Wahlbin, "Maximum norm estimates in the finite element method on plane polygonal domains. Part I," Math. Comp., v. 32, 1978, pp. 73-109.

8. R. ScotT, "Optimal $L^{\infty}$ estimates for the finite element method on irregular meshes," Math. Comp., v. 30,1976 , pp. 681-697.

9. D. JESPERSEN, "Ritz-Galerkin methods for singular boundary value problems," SIAM J. Numer. Anal., v. 15, 1978, pp. 813-834. 